\title{
Anabases
}

ANABASES Traditions et réceptions de l'Antiquité

$26 \mid 2017$

Varia

\section{PRISCIAN, Answers to King Khosroes of Persia}

\section{Olivier Gengler}

\section{OpenEdition}

\section{Journals}

Édition électronique

URL : http://journals.openedition.org/anabases/6268

DOI : 10.4000/anabases.6268

ISSN : 2256-9421

\section{Éditeur}

E.R.A.S.M.E.

\section{Édition imprimée}

Date de publication : 1 novembre 2017

Pagination : 245-247

ISSN : 1774-4296

\section{Référence électronique}

Olivier Gengler, « Priscian, Answers to King Khosroes of Persia », Anabases [En ligne], 26 | 2017, mis en ligne le 01 novembre 2017, consulté le 19 janvier 2021. URL : http://journals.openedition.org/ anabases/6268; DOI : https://doi.org/10.4000/anabases.6268

Ce document a été généré automatiquement le 19 janvier 2021.

(c) Anabases 


\title{
PRISCIAN, Answers to King Khosroes of Persia
}

\author{
Olivier Gengler
}

\section{RÉFÉRENCE}

PRISCIAN, Answers to King Khosroes of Persia, traduit par P. HUBY, S. EBBESEN, D. LANGSLOW, D. RUSSELL, C. STEEL et M. WILSON, Londres, Bloomsbury, 2016 (Ancient Commentators on Aristotle), VIII + 162 p., 90 livres / ISBN 978-1-4725-8413-7.

1 Philosophe actif dans le premier tiers $\mathrm{du} \mathrm{VI}^{\mathrm{e}} \mathrm{s}$. de notre ère, Priscien de Lydie est l'un des derniers représentants de la tradition néo-platonicienne non-chrétienne qui subsistait encore, à son époque, principalement à Alexandrie et à Athènes. L'ouvrage présenté ici donne la traduction anglaise de l'un des deux seuls textes connus de Priscien (auquel on attribue parfois aussi un commentaire au traité De l'âme d'Aristote transmis sous le nom de Simplicius), les Réponse à Chosroès (Solutiones ad Chosroem). Ce court texte constitué d'une préface et de dix chapitres, traduits ici par un groupe de spécialistes coordonné par R. Sorabji (Préface et ch.1:C.Steel, ch.2, 3, 4 et 6:D. Russel, ch.5 et 8 : S.Ebbesen, ch.7 : D.Russell et P. Huby, ch. $9:$ D. Langslow et S. Ebbesen, ch. 10 : M. Wilson, à partir d'une traduction littérale de l'ensemble par P. Huby) vient confirmer l'histoire fort singulière du voyage entrepris par sept philosophes grecs pour se rendre auprès du roi de perse Chosroès, qui avait la réputation d'être un véritable roi-philosophe platonicien, après que les mesures de Justinien contre les païens les avaient chassés de l'Empire romain (Agathias, Histoires, II, 30, 3 - 31, 9). Parmi ces sept philosophes, Damascius, alors scholarque de l'Académie, Simplicius et Priscien. L'épisode est habituellement mis en rapport avec la décision envoyée par Justinien à Athènes en 529 visant à interdire l'enseignement de la philosophie, comme le rapporte Jean Malalas dans sa Chronique (XVIII 47 379, 66 - 72 Thurn), et qui causa plus ou moins directement la fin de l'école néo-platonicienne athénienne. La question est plus complexe que ne le laisse entendre R. Sorabji dans l'introduction (p. 1-2), même si elle 
n'affecte que marginalement l'interprétation du texte de Priscien (une référence à E.J. Watts, City and School in Late Antique. Athens and Alexandria, Berkeley, 2006, 111-142, à compléter maintenant par la nouvelle version de l'article d'Alan Cameron, «The Last Days of the Academy at Athens», dansId., Wandering Poets and Other Essays on Late Greek Literature and Philosophy, Oxford, 2016, 205-245, aurait été nécessaire). Selon Agathias, les philosophes se seraient longuement entretenus avec le roi, mais auraient refusé de demeurer à sa cour, déçus par son érudition et effrayés par les mœurs perses. Il me semble cependant exagéré de voir dans les thèmes très naturalistes traités dans les Réponses la confirmation des attentes divergentes de Chosroès et de ses hôtes, comme le veut $\mathrm{R}$. Sorabji, qui semble par ailleurs persuadé que le roi a invité les philosophes à venir en Perse. S'il est possible que les Réponses soient, jusqu'à un certain point, un ouvrage de commande, il est contradictoire de donner une si grande initiative à Chosroès au vu du seul texte d'Agathias, qui contient d'ailleurs des marques manifestes d'ironie. Il manque par ailleurs dans l'introduction (p. 5) les références aux travaux de I. Hadot sur Simplicius auxquels il est pourtant fait référence (à savoir « La vie et l'œuvre de Simplicius", p. 21-22 et Simplicius. Commentaire sur le manuel d'Épictète. Introduction et édition critique du texte grec, Leiden, 1996, p. 24-28). Comme le rappelle justement R. Sorabji, I. Hadot y développe l'argument de M. Tardieu selon lequel les philosophes, au retour de Ctésiphon, se seraient établis à Harran: c'est là que Simplicius aurait écrit son commentaire au De caelo d'Aristote, comme en ferait foi l'évocation de bateaux particuliers, faits de peaux cousues et remplies d'air, qu'il n'aurait pu observer que lors de son voyage en Perse. À ce sujet, il faut ajouter les arguments développés par D. Marcotte, en dernier lieu dans un important article sur «Priscien de Lydie, la géographie et les origines néoplatoniciennes de la 'Collection philosophique' ", Journal des savants, 2014, p. 165-203.

2 Co-éditeur de la collection, R. Sorabji signe donc l'introduction générale du volume, ainsi que les introductions aux différents chapitres (mis à part le premier, présenté par C. Steel) et un certain nombre de notes. Celles-ci sont en effet également le fruit d'un travail collectif et, sauf quand elles sont de la main du ou des traducteurs du chapitre annoté, sont suivies des initiales du nom de leur auteur: outre les traducteurs euxmêmes, qui ont parfois annoté les traductions de leurs collègues, on trouve donc des notes de R. Sorabji, mais aussi de A.C. Bowen, S. White et V. Nutton. Ces notes portent essentiellement sur les choix de traduction et les équivalences latin-grec qui les justifient ainsi que des références aux textes parallèles. La principale difficulté posée par ce texte réside en effet dans le fait qu'il n'en subsiste qu'une version latine, œuvre d'un traducteur qui ne semble pas avoir totalement compris, ni le texte grec original, ni les arguments philosophiques et scientifiques qu'il exposait. La traduction du texte latin requiert donc un travail de rétroversion préalable pour surmonter plusieurs difficultés du texte et postuler, à partir d'une tournure latine obscure, quel pouvait être le texte grec sous-jacent. Ainsi, en 73, 3, où il est question des marées, il semble qu'il faille reconnaître le grec $\lambda$ óyọ derrière le latin verbum dans l'expression menstrualis verbi gratia et donc comprendre qu'il était question du « système mensuel » des marées, comparable au "système annuel » (annualis ratio) de 72, 2, comme le soulignait déjà Bywater dans son édition de référence du texte, qu'ont suivi les traducteurs (il faut corriger p. 149 et 156 la référence à cette occurrence de verbum dans les index : 73, 3 et non 73,2 , et fusionner les entrées verbum, logos et verbi gratia p. 156). Ce travail systématique donne des résultats convaincants. Suite à une proposition du découvreur du texte, J. Quicherat (la référence manque également: "Solution des problèmes 
proposés par Chosroès : traité inédit de Priscien le philosophe ", Bibliothèque de l'École des chartes, $3^{\mathrm{e}}$ série, 4, 1853,p. 248-263, aux p. 253-254), l'auteur de la traduction latine est parfois identifié à Jean Scot Érigène, une hypothèse que R. Sorabji (p. 6-7) préfère écarter, au vu de la piètre qualité du texte latin.

Les nombreuses notes qui accompagnent les chapitres traitent donc, pour l'essentiel, des questions posées par la traduction. Plus rarement, elles contiennent des éclaircissements sur le texte et des références bibliographiques. C'est plutôt dans les introductions particulières à chaque chapitre que le lecteur trouvera une rapide présentation des arguments philosophiques développés dans le texte. En fin de volume, outre un index des noms et des sujets très détaillé, compilé par R. Sorabji, se trouvent trois répertoires établis par D. Robertson (glossaire anglais-latin, index latin-anglais, index latin-grec) qui permettent d'explorer, à partir de la traduction, les principaux termes du texte original latin et leurs équivalents grecs supposés. Cette traduction rendra de grands services, en facilitant l'accès à un texte important et difficile. Elle constitue également une étape vers une nouvelle édition de l'original latin et, grâce à son appareil critique de notes et d'index, donne aux spécialistes tous les moyens de prolonger l'enquête.

\section{AUTEURS}

\section{OLIVIER GENGLER}

Académie des Sciences de Heidelberg

olivier.gengler@adw.uni-heidelberg.de 